\title{
SOME APPLICATIONS OF FOURIER ANALYSIS AND CAL- CULUS OF PROBABILITY TO THE STUDY OF REAL ROOTS OF ALGEBRAIC EQUATIONS $\left(^{(}\right)$
}

\author{
BY \\ ALFRED M. PElSER
}

\section{Introduction. Let}

$$
F=F(x, t)=F\left(x_{0}, \cdots, x_{n}, t\right)=x_{0}+x_{1} t+\cdots+x_{n} t^{n}=0
$$

be an algebraic equation with real coefficients, and let $N_{n}(x)=N_{n}\left(x_{0}, \cdots, x_{n}\right)$ denote the number of real roots of $(1.1)\left({ }^{2}\right)$. In this paper, we apply methods of Fourier analysis and calculus of probability to the study of the function $N_{n}\left(x_{0}, \cdots, x_{n}\right)$.

Let $K_{n}=\sum_{j=0}^{n} t^{2 i}, \quad L_{n}=\sum_{j=0}^{n} j t^{2 j-1}, \quad M_{n}=\sum_{j=0}^{n} j^{2} t^{2 j-2}, \quad D_{n}=K_{n} M_{n}-L_{n}^{2}$, $R_{n}=D_{n}^{1 / 2} / K_{n}$, and define two linear forms in $x_{0}, \cdots, x_{n}$ as follows: $X_{n}=\sum_{j=0}^{n} t^{i} x_{j} /\left(K_{n}^{1 / 2}\right), Y_{n}=\sum_{j=0}^{n}\left(K_{n} j t^{j-1}-L_{n} t^{j}\right) x_{j} /\left(K_{n} D_{n}\right)^{1 / 2}$. In $\S 4$ we show that at each continuity point of $N_{n}$,

$$
N_{n}(x)=\lim _{h \rightarrow \infty}\left(h /(2 \pi)^{1 / 2}\right) \int_{-\infty}^{\infty} R_{n}\left|Y_{n}\right| \exp \left[-X_{n}^{2} h^{2} / 2\right] d t .
$$

Now, $N_{n}(x)$ is an $(n+1)$-dimensional step function, and consideration of the discriminant of (1.1) shows that the set of points of discontinuity of $N_{n}$ (the set of points $\left(x_{0}, \cdots, x_{n}\right)$ for which $(1.1)$ has multiple roots) is an $(n+1)$ dimensional cone. Thus (1.2) defines $N_{n}(x)$ almost everywhere. We also notice that $d X_{n} / d t=R_{n} Y_{n}$.

To obtain (1.2), we start with a formula due to Kac [5, pp. 315-316](3):

$$
N_{n}(x)=\lim _{\epsilon \rightarrow 0}(1 / 2 \epsilon) \int_{-\infty}^{\infty} \psi_{\epsilon}(F)|\partial F / \partial t| d t,
$$

where $\psi_{\epsilon}(x)=1$ if $|x|<\epsilon$ and $\psi_{\epsilon}(x)=0$ otherwise. This formula holds for all choices of $x_{0}, \cdots, x_{n}$ if multiple roots are counted only once. In $\$ 2$, we develop a Fourier-like inversion formula for a certain class of step functions, and this formula, applied to (1.3), yields (1.2).

Other formulas for $N_{n}\left(x_{0}, \cdots, x_{n}\right)$, based on the expansion of (1.3) in an Hermite series, are also given in $\S 4$. In $\S 3$, we state without proof the lemmas

Presented to the Society, April 29,1944, under the title On the average sum of the real roots of a random algebraic equation, and February 26, 1944; received by the editors March 10, 1944 .

(1) The author is indebted to Professor Mark Kac for his aid in the preparation of this paper.

(2) For the case $F \equiv 0$, we define $N_{n}(0, \cdots, 0)=0$.

( $\left.{ }^{3}\right)$ Numbers in brackets refer to the references cited at the end of the paper. 
concerning Hermite series of functions of several variables which are needed in this connection.

It is easy to verify that $X_{n}$ and $Y_{n}$ are orthogonal, normal linear forms in $x_{0}, \cdots, x_{n}$. This fact is of particular importance if we assume that $x_{0}, \cdots, x_{n}$ are normally distributed independent random variables, so that $x_{j}$ has density

$$
\left(1 / s_{j}(2 \pi)^{1 / 2}\right) \exp \left[-\left(x-c_{j}\right)^{2} / 2 s_{j}^{2}\right] .
$$

By the additive property of the normal distribution $\left[2\right.$, p. 50], $X_{n}$ and $Y_{n}$ are themselves normally distributed, and the orthogonality implies that $X_{n}$ and $Y_{n}$ are mutually independent. Kac [6] considered the special case in which the $x$ 's all have standard normal distributions-that is, with $c_{j}=0$, $s_{j}=1$-and found that the mean value (mathematical expectation $=$ m.e.) of $N_{n}(x)$ is given by

$$
\text { m.e. }\left\{N_{n}(x)\right\}=(1 / \pi) \int_{-\infty}^{\infty} R_{n} d t \text {. }
$$

(The restriction $s_{j}=1$ is not essentially more severe than the restriction $s_{0}=s_{1}=\cdots=s_{n}$.) In $\$ 5$ we show that, because of the independence of $X_{n}$ and $Y_{n},(1.4)$ can be obtained easily from (1.2). Using (1.4), Kac also obtained the asymptotic formula

$$
\text { m.e. }\left\{N_{n}(x)\right\} \sim(2 / \pi) \log n .
$$

In $\S 6$, we consider the more general case in which the means $c_{j}$ may be different from zero and from each other. However, we still require that $s_{0}=s_{1}=\cdots=s_{n}=s$. This case may have an application to empirical equations; that is, equations in which the coefficients are to be determined experimentally. The observed coefficients can be written in the form $c_{j}+\epsilon_{j}$, where $c_{j}$ represents the "true" value, and $\epsilon_{j}$ represents the error term which may be assumed to be a normally distributed random variable with standard deviation $s$. We thus assume that all of the coefficients are measured with the same precision. With the further requirement that $\epsilon_{0}, \cdots, \epsilon_{n}$ be independent, we see that the "observed" coefficients are precisely of the type considered in $\S 6$. It would seem desirable to compare the average number of real roots of the "observed" equation with the number of real roots of the "true" equation

$$
c_{0}+c_{1} t+\cdots+c_{n} t^{n}=0 .
$$

In spite of the apparent simplicity of this problem, the computational diffculties seem enormous. Only in two cases did we succeed in obtaining reasonably accurate estimates of the difference between the average number of real roots of the "observed" equation and the number of real roots of the "true" equation. These two cases are:

I. The equation (1.6) has no real roots in the neighborhood of 1 and -1 . 
II. The equation (1.6) is such that there is an $\alpha_{n}>0$ such that $\left|c_{0}+c_{1} t+\cdots+c_{n} t^{n}\right|>\alpha_{n}, 0 \leqq t \leqq 1$.

$\$ 7$ is devoted primarily to the investigation of the average sum of real roots in a given interval. It is shown that the average sum of the absolute values of the real roots in an interval $(-A, A), A>1$, is asymptotically equal to the average number of real roots in this interval. This result makes even more apparent the fact already noticed by $\mathrm{Kac}[6, \mathrm{p} .320]$ that the real roots of random equations show a strong tendency to cluster (on the average) around 1 and -1 . Finally, we find that the average sum of the roots in an interval not containing 1 or -1 approaches a limit as $n \rightarrow \infty$. In fact, the average sum of the absolute values of the real roots in $(-a, a), 0<a<1$, approaches $-(1 / \pi) \log \left(1-a^{2}\right)$ as $n \rightarrow \infty$.

2. An inversion formula for step functions in more than one dimension. It is well known $[4$, p. 456] that if $f(x)$ is a one-dimensional step function, then for each continuity point of $f$,

$$
f(x)=\frac{e^{x^{2} / 2}}{2 \pi} \int_{-\infty}^{\infty} e^{i v x} d v \int_{-\infty}^{\infty} f(s) e^{-i v s-s^{2} / 2} d s .
$$

Thus, in considering an $(n+1)$-dimensional step function $f(x)=f\left(x_{0}, \cdots, x_{n}\right)$, one is naturally led to a generalization of this result which we can write formally as $\left({ }^{4}\right)$

$$
\begin{aligned}
f(x)=\frac{\exp \left(2^{-1} \sum x_{j}^{2}\right)}{(2 \pi)^{n+1}} & \int \cdots \int \exp \left(i \sum v_{j} x_{j}\right) d v \\
& \cdot \int \cdots \int f(s) \exp \left(-i \sum v_{j} s_{j}-2^{-1} \sum s_{j}^{2}\right) d s .
\end{aligned}
$$

In many cases, however, the right-hand side of the last equation is divergent, a fact which can be easily verified for the function $N_{n}(x)$. Therefore, following Hille [5, p. 448], we overcome this difficulty by introducing a parameter $z$ into the integrand. More precisely, for $0<z<1$, we write

$$
\begin{aligned}
P(f ; x, z)= & P\left(f ; x_{0}, \cdots, x_{n}, z\right) \\
= & \frac{\exp \left(2^{-1} \sum x_{j}^{2}\right)}{(2 \pi)^{n+1}} \\
& \cdot \int \cdots \int \exp \left(i \sum v_{j} x_{j}-2^{-1}\left(1-z^{2}\right) \sum v_{j}^{2}\right) d v \\
& \cdot \int \cdots \int f(s) \exp \left(-i z \sum v_{j} s_{j}-2^{-1} \sum s_{j}^{2}\right) d s .
\end{aligned}
$$

(4) Throughout this paper we shall use the symbol $\int \cdots \int d s$ to indicate integration over the entire $(n+1)$-space, and where no confusion arises, $\sum$ shall mean $\sum_{j=0}^{n}$. 
We see that, formally, $P(f ; x, 1)$ is exactly the integral which appears above, and in this section we shall show that for certain classes of step functions, $f(x)=\lim _{z \rightarrow 1}-P(f ; x, z)$ at every continuity point of $f$.

We begin with the simplest type of step function, namely the characteristic function of an $(n+1)$-dimensional open rectangle. Accordingly, let $T=E_{(x)}\left[a_{j}<x_{j}<b_{j} ; j=0,1, \cdots, n\right]$ and let $g(x)=g\left(x_{0}, \cdots, x_{n}\right)$ be the characteristic function of $T$. Obviously

$$
g(x)=\prod_{j=0}^{n} g_{j}\left(x_{j}\right)
$$

where

For $0<z<1$, let

$$
g_{j}(x)= \begin{cases}1, & a_{j}<x<b_{j} \\ 0, & \text { otherwise }\end{cases}
$$

$J(g ; x, z)=\frac{\exp \left(2^{-1} \sum x_{j}^{2}\right)}{\left(\left(2 \pi\left(1-z^{2}\right)\right)^{1 / 2}\right)^{n+1}}$

Using (2.3), we have

$$
\int \therefore \int g(s) \exp \left(-\frac{\sum\left(x_{i}-s_{j}\right)^{2}}{2\left(1-z^{2}\right)}-2^{-1} \sum s_{i}^{2}\right) d s \text {. }
$$

$$
J(g ; x, z)=\prod_{j=0}^{n} \frac{\exp \left(2^{-1} x_{j}^{2}\right)}{\left(2 \pi\left(1-z^{2}\right)\right)^{1 / 2}} \int_{-\infty}^{\infty} g_{j}(s) \exp \left(-\frac{\left(x_{j}-s\right)^{2}}{2\left(1-z^{2}\right)}-2^{-1} s^{2}\right) d s .
$$

Now, each integral on the right is the well known Weierstrass integral [3, p. 634] and we have

$$
g_{j}(x)=\lim _{z \rightarrow 1^{-}} \frac{\exp \left(2^{-1} x^{2}\right)}{\left(2 \pi\left(1-z^{2}\right)\right)^{1 / 2}} \int_{-\infty}^{\infty} g_{j}(s) \exp \left(-\frac{(x-s)^{2}}{2\left(1-z^{2}\right)}-2^{-1} s^{2}\right) d s
$$

at each continuity point of $g_{j}$. It follows at once that

$$
g(x)=\lim _{z \rightarrow 1^{-}} J(g ; x, z)
$$

at each continuity point of $g$.

LEMMA 2.1. If $f(x)$ is the characteristic function of an $(n+1)$-dimensional open set $R$, then at every continuity point of $f$,

$$
f(x)=\lim _{z \rightarrow 1^{-}} J(f ; x, z) .
$$

Proof. According to the definition of $f(x)$, 


$$
\begin{aligned}
& J(f ; x, z)=\frac{\exp \left(2^{-1} \sum x_{j}^{2}\right)}{\left(\left(2 \pi\left(1-z^{2}\right)\right)^{1 / 2}\right)^{n+1}} \\
& \cdot \int_{R} \cdots \int \exp \left(-\frac{\sum\left(x_{j}-s_{j}\right)^{2}}{2\left(1-z^{2}\right)}-2^{-1} \sum s_{i}^{2}\right) d s .
\end{aligned}
$$

Let $(x)$ be a continuity point of $f(x)$. If $(x) \notin R$, we can choose $\epsilon>0$ such that $\left|x_{j}-s_{j}\right|>\epsilon$ for at least one $j$. Thus

$$
|J(f ; x, z)| \leqq \frac{\exp \left(2^{-1} \sum x_{j}^{2}\right)}{\left(\left(1-z^{2}\right)^{1 / 2}\right)^{n+1}} \exp \left(-\epsilon^{2} / 2\left(1-z^{2}\right)\right),
$$

so that $\lim _{z \rightarrow 1^{-}} J(f ; x, z)=0$. If $(x) \in R$, then we choose an open $(n+1)$-dimensional rectangle $T$ with center $(x)$ and such that $T \subset R$. Thus, if $g(x)$ is the characteristic function of $T$, we have

$$
\begin{aligned}
J(f ; x, z)=J(g ; x, z)+ & \frac{\exp \left(2^{-1} \sum x_{j}^{2}\right)}{\left(\left(2 \pi\left(1-z^{2}\right)\right)^{1 / 2}\right)^{n+1}} \\
& \cdot \int_{R-T} \cdots \int \exp \left(-\frac{\sum\left(x_{j}-s_{j}\right)^{2}}{2\left(1-z^{2}\right)}-2^{-1} \sum s_{j}^{2}\right) d s .
\end{aligned}
$$

By an argument similar to that used above, the second member of the righthand side of this equation can be shown to approach zero as $z \rightarrow 1^{-}$, and using (2.4) we see that $\lim _{z \rightarrow 1^{-}}-J(f ; x, z)=1$. This proves the lemma.

We can now prove the main result of this section.

TheOREM 2.1. Let $R_{0}, \cdots, R_{p}$ be open sets such that the complement of $\sum_{j=0}^{p} R_{j}$ has $(n+1)$-dimensional Lebesgue measure zero. Let $f(x)=f\left(x_{0}, \cdots, x_{n}\right)$ be defined by

$$
f(x)=a_{i}, \quad(x) \in R_{j} .
$$

Then at each continuity point of $f, f(x)=\lim _{z \rightarrow 1}-P(f ; x, z)$.

Proof. There is clearly no loss of generality in supposing that $f(x)$ is the characteristic function of an open set. For $0<z<1$, the integrals in (2.2) are absolutely convergent, so we may interchange the order of integration and obtain by an easy computation

$$
\begin{aligned}
P(f ; x, z)= & \frac{\exp \left(2^{-1} \sum x_{j}^{2}\right)}{\left(\left(2 \pi\left(1-z^{2}\right)\right)^{1 / 2}\right)^{n+1}} \\
& \cdot \int \cdots \int f(s) \exp \left(-\frac{\sum\left(x_{j}-z s_{j}\right)^{2}}{2\left(1-z^{2}\right)}-2^{-1} \sum s_{j}^{2}\right) d s,
\end{aligned}
$$


so that $P(f ; x, z)=J(f ; x, z)+I(f ; x, z)$, where

$$
\begin{aligned}
I(f ; x, z)=\frac{\exp \left(2^{-1} \sum x_{j}^{2}\right)}{\left(\left(2 \pi\left(1-z^{2}\right)\right)^{1 / 2}\right)^{n+1}} & \\
\cdot \int \cdots \int f(s) \exp \left(-2^{-1} \sum s_{j}^{2}\right) & {\left[\exp \left(-\frac{\sum\left(x_{j}-z s_{j}\right)^{2}}{2\left(1-z^{2}\right)}\right)\right.} \\
& \left.-\exp \left(-\frac{\sum\left(x_{j}-s_{j}\right)^{2}}{2\left(1-z^{2}\right)}\right)\right] d s .
\end{aligned}
$$

If we let $\left(s_{j}-x_{j}\right) /\left(1-z^{2}\right)^{1 / 2}=v_{j}, j=0,1, \cdots, n$, and write

$G(s, x, z)=\exp \left(-2^{-1} \sum s_{j}^{2}\right)$

$$
\mid \exp \left(-2^{-1} \sum\left[s_{j}^{2}\left(z^{2}-1\right)-2 z x_{j} s_{j}\left(\frac{1-z}{1+z}\right)^{1 / 2}\right]\right)-1,
$$

we find that

$$
|I(f ; x, z)| \leqq \frac{\exp \left(2^{-1} \sum x_{j}^{2}\right)}{\left((2 \pi)^{1 / 2}\right)^{n+1}} \int \cdots \int G(s, x, z) d s .
$$

If $1 / 2^{1 / 2}<z<1$, then $\left(1-z^{2}\right)<1 / 2$ and $(1-z) /(i+z)<1 / 4$. Thus, for $1 / 2^{1 / 2}$ $<z<1, G(s, x, z)$ is dominated by

$$
\exp \left(-2^{-1} \sum s_{j}^{2}\right)\left[1+\exp \left(2^{-1} \sum\left|x_{j} s_{j}\right|+4^{-1} \sum s_{j}^{2}\right)\right]
$$

a clearly integrable function. It follows that, corresponding to an arbitrary $\epsilon>0$, we can choose an $(n+1)$-dimensional sphere $A$ with radius so large that, if $C(A)$ denotes the complement of $A$,

$$
\int_{C(A)} \cdots \int G(s, x, z) d s<\epsilon .
$$

Since $\lim _{z \rightarrow 1}-G(s, x, z)=0$ uniformly for $(s) \in A$,

$$
\int_{A} \cdots \int G(s, x, z) d s<\epsilon
$$

for $z$ sufficiently near 1 . Thus $\lim _{z \rightarrow 1^{-}}|I(f ; x, z)|=0$ and so $\lim _{z \rightarrow 1}-P(f ; x, z)$ $=\lim _{z \rightarrow 1^{-}} J(f ; x, z)$. The theorem follows by Lemma 2.1 .

In $\S 4$ we shall use Theorem 2.1 to obtain new formulas for $N_{n}(x)$. Before doing this, however, we first consider expansions in Hermite series of functions defined in more than one dimension, a subject closely related to the considerations of the present section. We shall then also obtain in $\$ 4$ an Hermite expansion of $N_{n}(x)$. 
3. Summability of Hermite series in more than one dimension. Hille [4, p. 450] has shown that for a function which satisfies fairly general conditions, the integral in (2.1) is summable, in the sense of Theorem 2.1 , to the same function to which the Hermite series of the given function is summable Abel. This result can be extended to the many-dimensional case. The properties of the Hermite polynomials which are needed in this connection are well known $[1$, pp. 331-351], and because of the similarity of the methods to those of $\$ 2$, the proofs will be omitted.

The $m$ th Hermite polynomial is defined by

$$
H_{m}(x)=(-1)^{m} \exp \left(2^{-1} x^{2}\right) d^{m} \exp \left(-2^{-1} x^{2}\right) / d x^{m} .
$$

For a function $g(x)=g\left(x_{0}, \cdots, x_{n}\right)$ satisfying the condition

$$
\int \cdots \int|g(x)|^{2} \exp \left(-2^{-1} \sum x_{j}^{2}\right) d x<\infty
$$

we write

$$
\begin{aligned}
A_{m_{0}} \cdots n_{n} & =\frac{1}{\left((2 \pi)^{1 / 2}\right)^{n+1}} \\
& \cdot \int \cdots \int g(s) \frac{H_{m_{0}}\left(s_{0}\right)}{m_{0} !} \cdots \frac{H_{m_{n}}\left(s_{n}\right)}{m_{n} !} \exp \left(-2^{-1} \sum s_{j}^{2}\right) d s,
\end{aligned}
$$

and

$$
S_{m}(g ; x)=S_{m}\left(g ; x_{0}, \cdots, x_{n}\right)=\sum_{\Sigma m j=m} A_{m_{0} \cdots m_{n}} H_{m_{0}}\left(x_{0}\right) \cdots H_{m_{n}}\left(x_{n}\right) .
$$

Recalling the notation of (2.2), we have the following theorem:

Theorem 3.1. If $g(x)=g\left(x_{0}, \cdots, x_{n}\right)$ satisfies (3.1), then for $0<z<1$, $P(g ; x, z)=\sum_{m=0}^{\infty} S_{m}(g ; x) z^{m}$.

The following theorem is an immediate corollary of Theorem 2.1 and Theorem 3.1.

TheOREM 3.2. If $f(x)$ is defined by (2.5), then at each continuity point of $f$, $f(x)=\lim _{z \rightarrow 1}-\sum_{m=0}^{\infty} S_{m}(f ; x) z^{m}$.

4. Formulas for $N_{n}(x)$. In this section we obtain several formulas for the number of real roots $N_{n}(x)=N_{n}\left(x_{0}, \cdots, x_{n}\right)$ of equation (1.1). Let us recall the following notation of $\S 1$ :

$$
\begin{aligned}
& K_{n}=\sum t^{2 j}, \quad L_{n}=\sum j t^{2 j-1}, \\
& M_{n}=\sum j^{2} t^{2 j-2}, \quad D_{n}=K_{n} M_{n}-L_{n}^{2} \text {, } \\
& R_{n}=D_{n}^{1 / 2} / K_{n} .
\end{aligned}
$$

Let $a_{j}=t^{j} / K_{n}^{1 / 2}$ and $b_{j}=\left(K_{n} j t^{i-1}-L_{n} t^{j}\right) /\left(K_{n} D_{n}\right)^{1 / 2}$, and let 


$$
\begin{aligned}
& X_{n}=X_{n}(x)=\sum a_{j} x_{j}, \\
& Y_{n}=Y_{n}(x)=\sum b_{j} x_{j} .
\end{aligned}
$$

Then we shall find that at each continuity point of $N_{n}$,

$$
N_{n}(x)=\lim _{n \rightarrow \infty}(1 / 2 \pi) \int_{-\infty}^{\infty} R_{n} d t
$$

and

$$
\int_{-\infty}^{\infty}|u| \exp \left[-\left(X_{n}^{2} h^{2}+\left(u-Y_{n} h\right)^{2}\right) / 2\right] d u
$$

$$
N_{n}(x)=\lim _{h \rightarrow \infty}\left(h /(2 \pi)^{1 / 2}\right) \int_{-\infty}^{\infty} R_{n}\left|Y_{n}\right| \exp \left[-X_{n}^{2} h^{2} / 2\right] d t .
$$

Other formulas for $N_{n}(x)-(4.11),(4.12)$ and (4.13)-will be presented later.

It is easy to verify that $\sum a_{j} b_{j}=0$ so that $X_{n}$ and $Y_{n}$ are orthogonal linear forms in $x_{0}, \cdots, x_{n}$; also that

$$
\sum a_{i}^{2}=\sum b_{j}^{2}=1 \text {. }
$$

These facts are of particular interest in the case that $x_{0}, \cdots, x_{n}$ are identical normally distributed independent random variables. For then the orthogonality of $X_{n}$ and $Y_{n}$ implies that $X_{n}$ and $Y_{n}$ are themselves normally distributed independent random variables, while if m.e. $\left\{x_{j}\right\}=0, j=0,1, \cdots, n$, then (4.5) means that $X_{n}$ and $Y_{n}$ have exactly the same distribution as have each of $x_{0}, \cdots, x_{n}$. A further interesting property of $X_{n}$ and $Y_{n}$, particularly for (4.4), is that

$$
d X_{n} / d t=R_{n} Y_{n} .
$$

To obtain our results, we start with Kac's formula (1.3). By virtue of (2.6),

$$
\begin{aligned}
& P\left(N_{n} ; x, z\right)= \frac{\exp \left(-\frac{z^{2} \sum x_{j}^{2}}{2\left(1-z^{2}\right)}\right)}{\left(\left(2 \pi\left(1-z^{2}\right)\right)^{1 / 2}\right)^{n+1}} \\
& \cdot \int \cdots \int N_{n}(u) \exp \left(\frac{z \sum x_{j} u_{j}}{1-z^{2}}-\frac{\sum u_{j}^{2}}{2\left(1-z^{2}\right)}\right) d u .
\end{aligned}
$$

Letting $u_{j}=v_{j}\left(1-z^{2}\right)^{1 / 2}$, and changing the order of integration,

$$
\begin{aligned}
& P\left(N_{n} ; x, z\right)=\exp \left(-\frac{z^{2} \sum x_{j}^{2}}{2\left(1-z^{2}\right)}\right) \int_{-\infty}^{\infty} d t \lim _{\epsilon \rightarrow 0} \frac{\left(1-z^{2}\right)^{1 / 2}}{2 \epsilon} \frac{1}{\left((2 \pi)^{1 / 2}\right)^{n+1}} \\
& \cdot \int \cdots \int \psi_{\epsilon}\left(\left(1-z^{2}\right)^{1 / 2} F(u, t)\right)|\partial F(u, t) / \partial t| \exp \left(\frac{z \sum x_{j} u_{j}}{\left(1-z^{2}\right)^{1 / 2}}-2^{-1} \sum u_{j}^{2}\right) d u .
\end{aligned}
$$


The change of order of integration is easily justified by means of the inequality $[5$, p. 316]

$$
(1 / 2 \epsilon) \int_{-\infty}^{\infty} \psi_{\epsilon}(F)|\partial F / \partial t| d t \leqq 3 n-5 .
$$

The integrals which appear above can best be handled by means of the formula

$$
\begin{aligned}
& \frac{1}{\left((2 \pi)^{1 / 2}\right)^{n+1}} \int \cdots \int\left[\prod_{r=1}^{k} f_{r}\left(\sum_{j=0}^{n} g_{r j} x_{j}\right)\right] \exp \left(-2^{-1} \sum_{j=0}^{n} x_{j}^{2}\right) d x \\
& =\frac{1}{(2 \pi)^{k / 2} \Delta_{k}^{1 / 2}} \int^{(k)} \cdots \int\left[\prod_{r=1}^{k} f_{r}\left(y_{r}\right)\right] \\
& \cdot \exp \left(-\frac{1}{2 \Delta_{k}} \sum_{r, s=1}^{k} \Delta_{k}^{r s} y_{r} y_{s}\right) d y_{1} \cdots d y_{k},
\end{aligned}
$$

where $\Delta_{k}=\left\|\sum_{j=0}^{n} q_{r j} q_{s j}\right\|, r, s=1,2, \cdots, k$, and where $\Delta_{k}^{r s}$ denotes the corresponding minor of $\Delta_{k}$. We assume, of course, that $\Delta_{k}>0$ and that $f_{1}, \cdots, f_{k}$ are such that the integrals exist. This formula can be obtained in many ways. Perhaps the best procedure is to use the language of probability [2, chaps. 6,10$]$. We can consider $x_{0}, \cdots, x_{n}$ as normally distributed independent random variables with mean zero and standard deviation one. Then $y_{r}=\sum_{j=0}^{n} q_{r j} x_{j}, r=1,2, \cdots, k$, are again normally distributed random variables $[2$, p. 50] and our formula follows from consideration of the joint distribution of $y_{1}, \cdots, y_{k}[2$, pp. 109-110].

If we put $k=3, q_{1 j}=t^{i}, q_{2 j}=j t^{i-1}$ and $q_{3 j}=x_{j}$, then

$$
\Delta_{3}=\left|\begin{array}{ccc}
K_{n} & L_{n} & \sum t^{j} x_{j} \\
L_{n} & M_{n} & \sum j t^{j-1} x_{j} \\
\sum t^{j} x_{j} & \sum j t^{j-1} x_{j} & \sum x_{j}^{2}
\end{array}\right|,
$$

and we find that

$$
P\left(N_{n} ; x, z\right)=\exp \left(-\frac{z^{2} \sum x_{i}^{2}}{2\left(1-z^{2}\right)}\right) \int_{-\infty}^{\infty} I_{n}(x, t) d t,
$$

where

$$
\begin{aligned}
& I_{n}(x, t)=\lim _{\epsilon \rightarrow 0} \frac{\left(1-z^{2}\right)^{1 / 2}}{2 \epsilon} \frac{1}{(2 \pi)^{3 / 2} \Delta_{3}^{1 / 2}} \\
& \quad \int_{-\infty}^{\infty} \int_{-\infty}^{\infty} \int_{-\infty}^{\infty} \psi_{\epsilon}\left(w\left(1-z^{2}\right)^{1 / 2}\right)|u| \exp \left(\frac{z v}{\left(1-z^{2}\right)^{1 / 2}}-\frac{\Delta(u, v, w)}{2 \Delta_{3}}\right) d u d v d w
\end{aligned}
$$

and 


$$
\Delta(u, v, w)=\Delta_{3}^{11} w^{2}+\Delta_{3}^{22} u^{2}+\Delta_{3}^{33} v^{2}+2 \Delta_{3}^{12} w u+2 \Delta_{3}^{13} w v+2 \Delta_{3}^{23} u v .
$$

According to the definition of $\psi_{\epsilon}$,

$$
\begin{aligned}
I_{n}(x, t) & =\frac{1}{(2 \pi)^{3 / 2} \Delta_{z}^{1 / 2}} \int_{-\infty}^{\infty} \int_{-\infty}^{\infty}|u| \exp \left(\frac{z v}{\left(1-z^{2}\right)^{1 / 2}}-\frac{\Delta(u, v, 0)}{2 \Delta_{3}}\right) d u d v \\
& =\frac{\exp \left(\Delta_{3}^{2} z / 2 D_{n}\left(1-z^{2}\right)\right)}{2 \pi D_{n}^{1 / 2}} \int_{-\infty}^{\infty}|\cdot u| \exp \left(-\frac{\Delta_{3}^{23} z u}{D_{n}\left(1-z^{2}\right)^{1 / 2}}-\frac{K_{n} \dot{u}^{2}}{2 D_{n}}\right) d u .
\end{aligned}
$$

We let $u=u^{\prime}\left(D_{n} / K_{n}\right)^{1 / 2}$, and noting that $\Delta_{3}^{23}=-Y_{n}\left(K_{n} D_{n}\right)^{1 / 2}$, we have

$I_{n}(x, t)=\frac{R_{n}}{2 \pi} \exp \left(-\frac{z^{2}\left(X_{n}^{2}-\sum x_{j}^{2}\right)}{2\left(1-z^{2}\right)}\right)$

Thus,

$$
\cdot \int_{-\infty}^{\infty}|u| \exp \left(-2^{-1}\left(u-z Y_{n} /\left(1-z^{2}\right)^{1 / 2}\right)^{2}\right) d u \text {. }
$$

$$
\begin{aligned}
P\left(N_{n} ; x, z\right)=\frac{1}{2 \pi} & \int_{-\infty}^{\infty} R_{n} d t \\
& \cdot \int_{-\infty}^{\infty}|u| \exp \left(-\frac{z^{2} X_{n}^{2}}{2\left(1-z^{2}\right)}-2^{-1}\left(u-z Y_{n} /\left(1-z^{2}\right)^{1 / 2}\right)^{2}\right) d u .
\end{aligned}
$$

Now $N_{n}(x)$ satisfies the conditions of Theorem 2.1, so equation (4.3) follows at once by putting $h=z /\left(1-z^{2}\right)^{1 / 2}$.

To derive (4.4), let $P_{n}(h)=P\left(N_{n} ; x, h /\left(h^{2}+1\right)^{1 / 2}\right)$, and let

$$
Q_{n}(h)=\left(h /(2 \pi)^{1 / 2}\right) \int_{-\infty}^{\infty} R_{n}\left|Y_{n}\right| \exp \left[-X_{n}^{2} h^{2} / 2\right] d t .
$$

Since $\lim _{h \rightarrow \infty} P_{n}(h)=N_{n}(x)$ at each continuity point of $N_{n}$, we show that $\lim _{h \rightarrow \infty}\left[P_{n}(h)-Q_{n}(h)\right]=0$. We have

$$
\begin{aligned}
P_{n}(h)-Q_{n}(h)= & \frac{1}{(2 \pi)^{1 / 2}} \int_{-\infty}^{\infty} R_{n} \exp \left[-X_{n}^{2} h^{2} / 2\right] \\
& \cdot\left[\frac{1}{(2 \pi)^{1 / 2}} \int_{-\infty}^{\infty}|u| \exp \left(-2^{-1}\left(u-h Y_{n}\right)^{2}\right) d u-h\left|Y_{n}\right|\right] d t .
\end{aligned}
$$

Using the well known formula

$$
|a|=\frac{1}{\pi} \int_{-\infty}^{\infty} \frac{1-\exp (i v a)}{v^{2}} d v,
$$

we obtain by a clearly justifiable change of order of integration 


$$
\begin{aligned}
\frac{1}{(2 \pi)^{1 / 2}} \int_{-\infty}^{\infty} \mid & u \mid \exp \left(-2^{-1}\left(u-h Y_{n}\right)^{2}\right) d u \\
& =\frac{1}{\pi(2 \pi)^{1 / 2}} \int_{-\infty}^{\infty} \frac{d v}{v^{2}} \int_{-\infty}^{\infty}(1-\exp (i v u)) \exp \left(-2^{-1}\left(u-h Y_{n}\right)^{2}\right) d u \\
& =\frac{1}{\pi} \int_{-\infty}^{\infty} \frac{1-\exp \left(i v h Y_{n}-2^{-1} v^{2}\right)}{v^{2}} d v
\end{aligned}
$$

and

Thus,

$$
h\left|Y_{n}\right|=\frac{1}{\pi} \int_{-\infty}^{\infty} \frac{1-\exp \left(i v h Y_{n}\right)}{v^{2}} d v .
$$

$$
\begin{aligned}
& P_{n}(h)-Q_{n}(h)=\frac{1}{\pi(2 \pi)^{1 / 2}} \int_{-\infty}^{\infty} R_{n} \exp \left[-X_{n}^{2} h^{2} / 2\right] d t \\
& \int_{-\infty}^{\infty} \exp \left(i v h Y_{n}\right) \frac{1-\exp \left(-2^{-1} v^{2}\right)}{v^{2}} d v .
\end{aligned}
$$

Since

$$
\begin{gathered}
\frac{1}{\pi} \int_{-\infty}^{\infty} \frac{1-\exp \left(-2^{-1} v^{2}\right)}{v^{2}} d v=\left(\frac{2}{\pi}\right)^{1 / 2}, \\
\left|P_{n}(h)-Q_{n}(h)\right| \leqq(1 / \pi) \int_{-\infty}^{\infty} R_{n} \exp \left[-X_{n}^{2} h^{2} / 2\right] d t .
\end{gathered}
$$

But $R_{n} \exp \left[-X_{n}^{2} h^{2} / 2\right]$ is dominated by $R_{n}$ which is integrable. Thus

$$
\lim _{h \rightarrow \infty} \int_{-\infty}^{\infty} R_{n} \exp \left[-X_{n}^{2} h^{2} / 2\right] d t=\int_{-\infty}^{\infty} R_{n} \lim _{h \rightarrow \infty} \exp \left[-X_{n}^{2} h^{2} / 2\right] d t=0 .
$$

This establishes (4.4).

We now obtain other formulas for $N_{n}(x)$, based on the results of $\S 3$. It can be shown, using (3.2), that $S_{2 m+1}\left(N_{n} ; x\right)=0$ and

$$
S_{2 m}\left(N_{n} ; x\right)=\frac{(-1)^{m+1}}{2^{m} \pi} \int_{-\infty}^{\infty} R_{n} \sum_{r=0}^{m} \frac{H_{2(m-r)}\left(X_{n}\right) H_{2 r}\left(Y_{n}\right)}{(m-r) ! r !(2 r-1)} d t .
$$

In obtaining (4.10), essentially the same integrals appear as those encountered in the derivation of (4.3). The only difficulties that arise are purely computational in nature and, accordingly, we shall omit the details. Now, $\sum_{m=0}^{\infty} S_{2 m}\left(N_{n} ; x\right)$ is a rearrangement of the formal Hermite series of $N_{n}(x)$, and therefore

$$
N_{n}(x)=\underset{r \rightarrow \infty}{\lim .} \sum_{m=0}^{r} S_{2 m}\left(N_{n} ; x\right) \text {. }
$$

Let $(x)$ be a continuity point of $N_{n}$. Since $N_{n}$ has the form (2.5), we can choose a set $T_{x}$ such that $(x) \in T_{x}$ and $N_{n}$ is constant over $T_{x}$. Then if $\left|T_{x}\right|$ 
denotes the $(n+1)$-dimensional Lebesgue measure of $T_{x}$,

$$
\int_{T_{x}} \cdots \int N_{n}(s) d s=N_{n}(x)\left|T_{x}\right|
$$

Thus, termwise integration of the formal Hermite series yields

$$
N_{n}(x)=\frac{1}{\left|T_{x}\right|} \sum_{m=0}^{\infty} \int_{T_{x}} \ldots \int S_{2 m}\left(N_{n} ; s\right) d s .
$$

If the diameter of $T_{x}$ is sufficiently small, we can choose $T_{x}$ in any manner that we please, and it is conceivable that for some particular choices of $x_{0}, \cdots, x_{n}, T_{x}$ can be taken in such a way as to make (4.12) useful.

Finally, in light of (4.10) and Theorem 3.2, we have at each continuity point of $N_{n}$,

$$
N_{n}(x)=\lim _{z \rightarrow 1^{-}} \sum_{m=0}^{\infty} \frac{(-1)^{m+1} z^{2 m}}{2^{m} \pi} \int_{-\infty}^{\infty} R_{n} \sum_{r=0}^{m} \frac{H_{2(m-r)}\left(X_{n}\right) H_{2 r}\left(Y_{n}\right)}{(m-r) ! r !(2 r-1)} d t .
$$

5. A formula for m.e. $\left\{N_{n}(x)\right\}$. We suppose now that the coefficients $x_{0}, \cdots, x_{n}$ in equation (1.1) are independent random variables. The results of this section are similar to those obtained by Kac [5], and we follow the methods which are employed there. We need the following lemma.

LEMMA 5.1. For each $h>0$,

$$
\left(h /(2 \pi)^{1 / 2}\right) \int_{-\infty}^{\infty} R_{n}\left|Y_{n}\right| \exp \left[-X_{n}^{2} h^{2} / 2\right] d t<3 n-1 .
$$

Proof. Let $t_{1}, \cdots, t_{p}$ denote the points at which $X_{n}$ has a relative maximum or minimum. If $v_{k}=\left.X_{n}\right|_{t-t_{k}}$,

$$
\begin{aligned}
\left(h /(2 \pi)^{1 / 2}\right) \int_{t_{k}}^{t_{k+1}} R_{n}\left|Y_{n}\right| \exp \left[-X_{n}^{2} h^{2} / 2\right] d t & \\
& =\left(h /(2 \pi)^{1 / 2}\right)\left|\int_{v_{k}}^{v_{k+1}} \exp \left[-u^{2} h^{2} / 2\right] d u\right|<1
\end{aligned}
$$

using (4.6). Also,

$$
\begin{aligned}
\left(h /(2 \pi)^{1 / 2}\right) \int_{-\infty}^{t_{1}} R_{n}\left|Y_{n}\right| \exp \left[-X_{n}^{2} h^{2} / 2\right] d t & \\
& \leqq\left(h /(2 \pi)^{1 / 2}\right) \int_{-\infty}^{\left|v_{1}\right|} \exp \left[-u^{2} h^{2} / 2\right] d u<1,
\end{aligned}
$$

and similarly

$$
\left(h /(2 \pi)^{1 / 2}\right) \int_{t_{p}}^{\infty} R_{n}\left|Y_{n}\right| \exp \left[-X_{n}^{2} h^{2} / 2\right] d t<1 .
$$


The lemma follows from the remark that $p$ is not greater than the highest power of $t$ in the numerator of $Y_{n}$; that is, $p \leqq 3 n-2$.

THEOREM 5.1. If $x_{0}, \cdots, x_{n}$ are independent random variables with finite first absolute moments, then

(5.1) m.e. $\left\{N_{n}(x)\right\}=\lim _{h \rightarrow \infty}\left(h /(2 \pi)^{1 / 2}\right) \int_{-\infty}^{\infty} R_{n}$ m.e. $\left\{\left|Y_{n}\right| \exp \left[-X_{n}^{2} h^{2} / 2\right]\right\} d t$.

Proof. Since mathematical expectation is nothing but a Lebesgue integral over a set with a completely additive Lebesgue measure, the measure of the whole set being one, it follows from Lemma 5.1 and a well known theorem from Lebesgue's theory that

$$
\text { m.e. }\left\{N_{n}(x)\right\}=\lim _{h \rightarrow \infty}\left(h /(2 \pi)^{1 / 2}\right) \text { m.e. }\left\{\int_{-\infty}^{\infty} R_{n}\left|Y_{n}\right| \exp \left[-X_{n}^{2} h^{2} / 2\right] d t\right\} \text {. }
$$

Using (4.2), we see that

$$
\int_{-\infty}^{\infty} R_{n} \text { m.e. }\left\{\left|Y_{n}\right| \exp \left[-X_{n}^{2} h^{2} / 2\right]\right\} d t<\max _{0 \leqq j \leqq n} \text { m.e. }\left\{\left|x_{j}\right|\right\} \int_{-\infty}^{\infty} R_{n} \sum\left|b_{j}\right| d t .
$$

An examination of $b_{j}$ shows that this last integral exists. Thus

$$
\text { m.e. }\left\{\int_{-\infty}^{\infty} R_{n}\left|Y_{n}\right| \exp \left[-X_{n}^{2} h^{2} / 2\right] d t\right\}
$$

and the theorem follows.

$$
=\int_{-\infty}^{\infty} R_{n} \text { m.e. }\left\{\left|Y_{n}\right| \exp \left[-X_{n}^{2} h^{2} / 2\right]\right\} d t
$$

In case $x_{0}, \cdots, x_{n}$ are normally distributed, the orthogonality of $X_{n}$ and $Y_{n}$, as pointed out above, implies their independence. Thus, from a well known property of independent random variables, we have Theorem 5.2.

THEOREM 5.2. If $x_{0}, \cdots, x_{n}$ are independent, normally distributed random variables, then

$$
\text { m.e. }\left\{N_{n}(x)\right\}
$$

$$
=\lim _{h \rightarrow \infty}\left(h /(2 \pi)^{1 / 2}\right) \int_{-\infty}^{\infty} R_{n} \text { m.e. }\left\{\left|Y_{n}\right|\right\} \text { m.e. }\left\{\exp \left[-X_{n}^{2} h^{2} / 2\right]\right\} d t .
$$

The formula (1.4) obtained by $\mathrm{Kac}$ is now a simple consequence of Theorem 5.2 and (4.5). For if $x_{0}, \cdots, x_{n}$ have independent, standard normal distributions, then so have $X_{n}$ and $Y_{n}$, whence

$$
\text { m.e. }\left\{\left|Y_{n}\right|\right\}=\frac{1}{(2 \pi)^{1 / 2}} \int_{-\infty}^{\infty}|s| \exp \left(-2^{-1} s^{2}\right) d s=\left(\frac{2}{\pi}\right)^{1 / 2}
$$


and

m.e. $\left\{\exp \left[-X_{n}^{2} h^{2} / 2\right]\right\}=\frac{1}{(2 \pi)^{1 / 2}} \int_{-\infty}^{\infty} \exp \left(-2^{-1} h^{2} s^{2}-2^{-1} s^{2}\right) d s=\frac{1}{\left(h^{2}+1\right)^{1 / 2}}$, so that

$$
\text { m.e. }\left\{N_{n}(x)\right\}=\lim _{h \rightarrow \infty} \frac{h}{\pi\left(h^{2}+1\right)^{1 / 2}} \int_{-\infty}^{\infty} R_{n} d t=\frac{1}{\pi} \int_{-\infty}^{\infty} R_{n} d t .
$$

6. The average number of real roots in case the coefficients have means different from zero. Remarks on empirical equations. In this section we suppose that the coefficients in (1.1) are independent, normally distributed random variables with different means, say $c_{0}, \cdots, c_{n}$, but having the same standard deviation $s$. Thus, if $x_{0}, \cdots, x_{n}$ are independent, standard normally distributed random variables, we can write (1.1) in the form

$$
\left(c_{0}+s x_{0}\right)+\left(c_{1}+s x_{1}\right) t+\cdots+\left(c_{n}+s x_{n}\right) t^{n}=0 .
$$

Writing $N_{n}(c+s x)=N_{n}\left(c_{0}+s x_{0}, \cdots, c_{n}+s x_{n}\right)$, we find from (5.2) that the average number of real roots of $(6.1)$ is

m.e. $\left\{N_{n}(c+s x)\right\}=\lim _{h \rightarrow \infty}\left(h /(2 \pi)^{1 / 2}\right) \int_{-\infty}^{\infty} R_{n}$ m.e. $\left\{\left|Y_{n}(c+s x)\right|\right\}$

Now,

$$
\text { m.e. }\left\{\exp \left[-X_{n}^{2}(c+s x) h^{2} / 2\right]\right\} d t \text {. }
$$

$$
\text { m.e. } \begin{aligned}
\left\{\left|Y_{n}(c+s x)\right|\right\} & =\text { m.e. }\left\{\left|Y_{n}(c)+s Y_{n}(x)\right|\right\} \\
& =\left(s /(2 \pi)^{1 / 2}\right) \int_{-\infty}^{\infty}|y| \exp \left[-\left(y-Y_{n}(c) / s\right)^{2} / 2\right] d y
\end{aligned}
$$

and

$$
\begin{aligned}
\text { m.e. }\left\{\exp \left[-X_{n}^{2}(c+s x) h^{2} / 2\right]\right\} & =\text { m.e. }\left\{\exp \left[-\left(X_{n}(c)+s X_{n}(x)\right)^{2} h^{2} / 2\right]\right\} \\
= & \left(1 /\left(h^{2} s^{2}+1\right)^{1 / 2}\right) \exp \left[-X_{n}^{2}(c) h^{2} / 2\left(h^{2} s^{2}+1\right)\right] .
\end{aligned}
$$

Therefore

$$
\text { m.e. }\left\{N_{n}(c+s x)\right\}=(1 / 2 \pi) \int_{-\infty}^{\infty} R_{n} \exp \left[-X_{n}^{2}(c) / 2 s^{2}\right] d t
$$

$$
\int_{-\infty}^{\infty}|y| \exp \left[-\left(y-Y_{n}(c) / s\right)^{2} / 2\right] d y .
$$

Let $\epsilon_{n}>0$ be chosen so small that

$$
P_{\epsilon_{n}}=E_{(t)}\left[\left|X_{n}(c)\right|<\epsilon_{n}\right]
$$

contains no turning points of $X_{n}(c)$ which are not zeros of $X_{n}(c)$. In case $c_{n} \neq 0$, we can write 


$$
P_{e_{n}}=\sum_{j=1}^{N_{n}(c)} I_{j}
$$

where the $I$ 's are disjoint open intervals, say $\left(r_{j}, s_{j}\right), j=1,2, \cdots, N_{n}(c)$, and where $N_{n}(c)$ represents, of course, the number of real roots of

$$
c_{0}+c_{1} t+\cdots+c_{n} t^{n}=0 .
$$

If $c_{n}=0$, then $P_{\epsilon_{n}}=\sum I_{j}+(-\infty, a)+(b,+\infty)$, where $a$ and $b$ are suitably chosen constants. We shall suppose that $c_{n} \neq 0$; that is, that (6.4) holds. If $c_{n}=0$; the arguments below still hold with slight modification. We use $C\left(P_{\epsilon_{n}}\right)$ to denote the complement of $P_{e_{n}}$. Let

$$
A_{n}(c, v)=\left(1 / v(2 \pi)^{1 / 2}\right) \int_{-\infty}^{\infty} R_{n}\left|Y_{n}(c)\right| \exp \left[-X_{n}^{2}(c) / 2 v^{2}\right] d t
$$

and

$$
B_{n}\left(c, v, \epsilon_{n}\right)=\left(1 / v(2 \pi)^{1 / 2}\right) \int_{P_{\epsilon_{n}}} R_{n}\left|Y_{n}(c)\right| \exp \left[-X_{n}^{2}(c) / 2 v^{2}\right] d t
$$

An immediate consequence of (4.4) is that $N_{n}(c)=\lim _{v \rightarrow 0} A_{n}(c, v)$. Since

$$
A_{n}(c, v)-B_{n}\left(c, v, \epsilon_{n}\right)
$$

$$
=\left(1 / v(2 \pi)^{1 / 2}\right) \int_{C\left(P_{\left.e_{n}\right)}\right)} R_{n}\left|Y_{n}(c)\right| \exp \left[-X_{n}^{2}(c) / 2 v^{2}\right] d t,
$$

we have

$$
\left|A_{n}(c, v)-B_{n}\left(c, v, \epsilon_{n}\right)\right| \leqq\left(1 / v(2 \pi)^{1 / 2}\right) \exp \left[-\epsilon_{n}^{2} / 2 v^{2}\right] \int_{-\infty}^{\infty} R_{n}\left|Y_{n}(c)\right| d t .
$$

Thus, $\lim _{v \rightarrow 0}\left[A_{n}(c, v)-B_{n}\left(c, v, \epsilon_{n}\right)\right]=0$, and therefore

$$
N_{n}(c)=\lim _{v \rightarrow 0} B_{n}\left(c, v, \epsilon_{n}\right) .
$$

By (4.9), we see that

$$
\mid \text { m.e. }\left\{\dot{N}_{n}(c+s x)\right\}-A_{n}(c, s) \mid \leqq(1 / \pi) \int_{-\infty}^{\infty} R_{n} \exp \left[-X_{n}^{2}(c) / 2 s^{2}\right] d t .
$$

We now proceed to show that

$$
N_{n}(c)-B_{n}\left(c, s, \epsilon_{n}\right)=(2 / \pi)^{1 / 2} N_{n}(c) \int_{\epsilon_{n / s}}^{\infty} \exp \left(-2^{-1} u^{2}\right) d u .
$$

Since 


$$
\begin{aligned}
& \int_{1 / \bullet}^{1 / v}\left(1-u^{2} X_{n}^{2}\right) \exp \left[-X_{n}^{2} u^{2} / 2\right] d u \\
& \quad=(1 / v) \exp \left[-X_{n}^{2} / 2 v^{2}\right]-(1 / s) \exp \left[-X_{n}^{2} / 2 s^{2}\right],
\end{aligned}
$$

we have

$$
\begin{aligned}
& B_{n}\left(c, v, \epsilon_{n}\right)-B_{n}\left(c, s, \epsilon_{n}\right) \\
& \quad=\left(1 /(2 \pi)^{1 / 2}\right) \int_{P_{\epsilon_{n}}} R_{n}\left|Y_{n}(c)\right| d t \int_{1 / 8}^{1 / v}\left(1-u^{2} X_{n}^{2}(c)\right) \exp \left[-X_{n}^{2}(c) u^{2} / 2\right] d u .
\end{aligned}
$$

Now, using (4.6), we can integrate by parts and obtain

$$
\begin{aligned}
& \int_{I_{j}} R_{n}\left|Y_{n}(c)\right| u^{2} X_{n}^{2}(c) \exp \left[-X_{n}^{2}(c) u^{2} / 2\right] d t \\
& \left.\quad=-X_{n}(c) \exp \left[-X_{n}^{2}(c) u^{2} / 2\right]\right]_{r_{j}}^{s j}+\int_{I_{j}} R_{n}\left|Y_{n}(c)\right| \exp \left[-X_{n}^{2}(c) u^{2} / 2\right] d t .
\end{aligned}
$$

Since

$$
\left.X_{n}(c) \exp \left[-X_{n}^{2}(c) u^{2} / 2\right]\right]_{r j}^{s i}=2 \epsilon_{n} \exp \left[-\epsilon_{n}^{2} u^{2} / 2\right]
$$

we find, using (6.4), that

$$
\begin{aligned}
& \int_{P_{\epsilon_{n}}} R_{n}\left|Y_{n}(c)\right| u^{2} X_{n}^{2}(c) \exp \left[-X_{n}^{2}(c) u^{2} / 2\right] d t \\
& \quad=-2 \epsilon_{n} N_{n}(c) \exp \left[-\epsilon_{n}^{2} u^{2} / 2\right]+\int_{P_{\epsilon_{n}}} R_{n}\left|Y_{n}(c)\right| \exp \left[-X_{n}^{2}(c) u^{2} / 2\right] d t,
\end{aligned}
$$

so that

$$
B_{n}\left(c, v, \epsilon_{n}\right)-B_{n}\left(c, s, \epsilon_{n}\right)=(2 / \pi)^{1 / 2} \epsilon_{n} N_{n}(c) \int_{1 / \varepsilon}^{1 / v} \exp \left(-2^{-1} \epsilon_{n}^{2} u^{2}\right) d u .
$$

Formula (6.11) is now seen to follow from (6.9) if we let $v \rightarrow 0$.

Using (6.8), (6.10), (6.11) and the well known inequality

$$
\int_{a}^{\infty} \exp \left(-2^{-1} u^{2}\right) d u<(\pi / 2)^{1 / 2} \exp \left(-2^{-1} a^{2}\right), \quad a>0
$$

we have

$$
\begin{aligned}
& \mid N_{n}(c)-\text { m.e. }\left\{N_{n}(c+s x)\right\} \mid \\
& <(1 / \pi) \int_{-\infty}^{\infty} R_{n} \exp \left[-X_{n}^{2}(c) / 2 s^{2}\right] d t \\
& \quad+\left(1 / s(2 \pi)^{1 / 2}\right) \int_{c\left(P_{e_{n}}\right)} R_{n}\left|Y_{n}(c)\right| \exp \left[-X_{n}^{2}(c) / 2 s^{2}\right] d t \\
& \quad+N_{n}(c) \exp \left[-\epsilon_{n}^{2} / 2 s^{2}\right] .
\end{aligned}
$$


It is interesting to ask under what conditions the left-hand side of (6.12) is near zero. That some condition must be placed on the polynomial in (6.5) is apparent from consideration of the trivial case $c_{0}=\cdots=c_{n}=0$; for then, by (1.5),

$$
\mid N_{n}(c) \text { - m.e. }\left\{N_{n}(c+s x)\right\} \mid=\text { m.e. }\left\{N_{n}(s x)\right\} \sim(2 / \pi) \log n .
$$

We shall, in the following, exclude this case from consideration, and also exclude those polynomials whose real zeros are near 1 and -1 .

THEOREM 6.1. If, for a fixed $\beta, 0<\beta<1$, equation (6.5) has no real roots in $1-\beta<|t|<1+\beta$, and if $s=\epsilon_{n} /\left(2 a_{n} \log n\right)^{1 / 2}$, where $a_{n}>k>1$, then, for $n \geqq 2$,

$$
\mid N_{n}(c) \text { - m.e. }\left\{N_{n}(c+s x)\right\} \mid<\frac{5}{n^{k-1}}+\frac{4\left|P_{\epsilon_{n}}\right|}{\pi \beta\left(4-\beta^{2}\right)},
$$

where $\left|P_{\epsilon_{n}}\right|$ denotes the Lebesgue measure of $P_{\epsilon_{n}}$.

In order to prove Theorem 6.1, we shall need the following lemma.

Lemma 6.1. For $s>0$,

$$
\begin{aligned}
\left(1 / s(2 \pi)^{1 / 2}\right) \int_{C\left(P_{\left.\epsilon_{n}\right)}\right.} R_{n}\left|Y_{n}(c)\right| \exp [- & \left.X_{n}^{2}(c) / 2 s^{2}\right] d t \\
& <\left(8 n s / \epsilon_{n}(2 \pi)^{1 / 2}\right) \exp \left(-\epsilon_{n}^{2} / 2 s^{2}\right) .
\end{aligned}
$$

Proof. The left-hand side of (6.14) is certainly less than

$$
A=\left(1 / s \epsilon_{n}(2 \pi)^{1 / 2}\right) \int_{C\left(P_{\epsilon_{n}}\right)} R_{n}\left|Y_{n}(c) X_{n}(c)\right| \exp \left[-X_{n}^{2}(c) / 2 s^{2}\right] d t .
$$

Let $t_{1}, \cdots, t_{p}$ denote the points at which $X_{n}(c)$ has a relative maximum or minimum but which are not zeros of $X_{n}(c)$. Using the notation of (6.4), we arrange the $r_{j}, s_{j}$, and $t_{j}$ in ascending order and integrate over the successive intervals so formed, omitting, of course, the intervals $\left(r_{j}, s_{j}\right)$. We find integrals of the following kinds:

$$
\begin{aligned}
\left(1 / s \epsilon_{n}\right) \int_{s_{j}}^{t_{k}} R_{n}\left|Y_{n}(c) X_{n}(c)\right| \exp & {\left[-X_{n}^{2}(c) / 2 s^{2}\right] d t } \\
= & \pm\left(s / \epsilon_{n}\right)\left[\exp \left[-X_{n}^{2}(c) / 2 s^{2}\right]\right]_{s j}^{t k} \\
& = \pm\left(s / \epsilon_{n}\right)\left[\exp \left[-v_{k}^{2} / 2 s^{2}\right]-\exp \left[-\epsilon_{n}^{2} / 2 s^{2}\right]\right]
\end{aligned}
$$

where $v_{k}=\left.X_{n}(c)\right|_{t=t_{k}}$;

$$
\begin{aligned}
\left(1 / s \epsilon_{n}\right) \int_{t_{k}}^{t_{k+1}} R_{n}\left|Y_{n}(c) X_{n}(c)\right| & \exp \left[-X_{n}^{2}(c) / 2 s^{2}\right] d t \\
= & \pm\left(s / \epsilon_{n}\right)\left[\exp \left[-v_{k+1}^{2} / 2 s^{2}\right]-\exp \left[-v_{k}^{2} / 2 s^{2}\right]\right]
\end{aligned}
$$




$$
\begin{aligned}
\left(1 / s \epsilon_{n}\right) \int_{t_{k}}^{r_{j}} R_{n}\left|Y_{n}(c) X_{n}(c)\right| \exp & {\left[-X_{n}^{2}(c) / 2 s^{2}\right] d t } \\
= & \pm\left(s / \epsilon_{n}\right)\left[\exp \left[-\epsilon_{n}^{2} / 2 s^{2}\right]-\exp \left[-v_{k}^{2} / 2 s^{2}\right]\right] .
\end{aligned}
$$

In each of these integrals we have made use of (6.4). Since the choice of $\epsilon_{n}$ implies that $\left|v_{k}\right|>\epsilon_{n}$, each of the above integrals is certainly less than $\left(2 s / \epsilon_{n}\right) \exp \left[-\epsilon_{n}^{2} / 2 s^{2}\right]$. We also need to consider integrals from $-\infty$ to the smallest of the $r_{j}, s_{j}, t_{j}$ and from the largest of these to $+\infty$. It is easy to see that the last estimate holds for these integrals as well. Now $p$, the number of relative maxima and minima of $X_{n}(c)$, is certainly not greater than the highest power of $t$ in the numerator of $Y_{n}(c)$; that is, $p \leqq 3 n-2$. Thus the number of integrals involved above is less than $4 n$. Hence $A \leqq\left(8 n s / \epsilon_{n}(2 \pi)^{1 / 2}\right)$ $\cdot \exp \left[-\epsilon_{n}^{2} / 2 s^{2}\right]$. This proves the lemma.

Proof of Theorem 6.1. Denote by $J_{1}, J_{2}, J_{3}$ the three terms on the righthand side of (6.12). From the inequality $[5$, p. 319]

$$
R_{n} \leqq \frac{1}{\left|1-t^{2}\right|}
$$

and the hypothesis of the theorem, we see that if $S=E_{(t)}[|t| \leqq 1]$ and $T=E_{(t)}[|t|>1]$, then

$$
\begin{aligned}
(1 / \pi) \int_{P_{\epsilon_{n}}} R_{n} \exp [ & \left.-X_{n}^{2}(c) / 2 s^{2}\right] d t \\
& \leqq(1 / \pi) \int_{S P_{\epsilon_{n}}} \frac{d t}{1-t^{2}}+(1 / \pi) \int_{T P_{\epsilon_{n}}} \frac{d t}{t^{2}-1}<\frac{4\left|P_{\epsilon_{n}}\right|}{\pi \beta\left(4-\beta^{2}\right)} .
\end{aligned}
$$

Also, since [5, p. 319]

$$
(1 / \pi) \int_{-\infty}^{\infty} R_{n} d t<(2 / \pi) \log n+(14 / \pi)
$$

we have

Thus

$$
\begin{aligned}
(1 / \pi) \int_{C\left(P_{\epsilon_{n}}\right)} R_{n} \exp \left[-X_{n}^{2}(c) / 2 s^{2}\right] d t & <(1 / \pi) \exp \left[-\epsilon_{n}^{2} / 2 s^{2}\right] \int_{-\infty}^{\infty} R_{n} d t \\
& <(14+2 \log n) / n^{k} .
\end{aligned}
$$

$$
J_{1}<(14+2 \log n) / \pi n^{k}+4\left|P_{\epsilon_{n}}\right| / \pi \beta\left(4-\beta^{2}\right) .
$$

Using Lemma 6.1, we find that

$$
\begin{aligned}
J_{2}+J_{3} & <\exp \left[-\epsilon_{n}^{2} / 2 s^{2}\right]\left(8 n s / \epsilon_{n}(2 \pi)^{1 / 2}+N_{n}(c)\right) \\
& <\left(4 /(\pi \log n)^{1 / 2}+1\right) / n^{k-1} .
\end{aligned}
$$

Combining (6.17) and (6.18) and supposing $n>2$, we obtain (6.13). 
The question of the proper order of $s / \epsilon_{n}$ to insure that m.e. $\left\{N_{n}(c+s x)\right\}$ is near $N_{n}(c)$ is a delicate one and we shall attempt to answer it in only one particular case. To that end, we shall confine ourselves to the interval $0 \leqq t \leqq 1$, and $N_{n}(c)$ will designate the number of roots of (6.5) in this interval. All previous results hold in this interval by simply replacing the integrals over $(-\infty, \infty)$ with respect to $t$ by integrals over $(0,1)$. We assume furthermore that the polynomials in (6.5) are bounded away from zero uniformly for all $n$; that is, there is an $\epsilon>0$ such that for $0 \leqq t \leqq 1$,

$$
\left|\sum_{j=0}^{n} c_{j} t^{i}\right|>\epsilon, \quad n=1,2, \cdots
$$

A trivial consequence of $(6.19)$ is that $N_{n}(c)=0$.

LEMMA 6.2. If

preal, then

$$
I(p)=\int_{-\infty}^{\infty}|y| \exp \left[-(y-p)^{2} / 2\right] d y,
$$

$$
2 \leqq I(p) \leqq 2+(2 \pi)^{1 / 2}|p| .
$$

Proof. Formula (4.7) and an easily justified change of order of integration yield

Let

$$
\begin{aligned}
I(p)= & (2 / \pi)^{1 / 2} \int_{-\infty}^{\infty}\left(1-(\cos p w) \exp \left[-w^{2} / 2\right]\right) d w / w^{2} \\
= & (2 / \pi)^{1 / 2} \int_{-\infty}^{\infty}\left(1-\exp \left[-w^{2} / 2\right]\right) d w / w^{2} \\
& +(2 / \pi)^{1 / 2} \int_{-\infty}^{\infty}(1-\cos p w) \exp \left[-w^{2} / 2\right] d w / w^{2}
\end{aligned}
$$

Then

$$
J(g)=\int_{-\infty}^{\infty}(1-\cos w) \exp \left[-w^{2} / 2 q\right] d w / w^{2}, \quad g>0
$$

$$
\begin{aligned}
J^{\prime}(g) & =\left(1 / 2 q^{2}\right) \int_{-\infty}^{\infty}(1-\cos w) \exp \left[-w^{2} / 2 q\right] d w \\
& =\left(\pi / 2 q^{3}\right)^{1 / 2}(1-\exp [-q / 2]) .
\end{aligned}
$$

Since $J(q)$ is a continuous function of $q$, and since $J(0)=0$,

$$
J(q)=\int_{0}^{q} J^{\prime}(s) d s=(\pi / 2 d)^{1 / 2} \int_{0}^{q}(1-\exp [-s / 2]) d s / s^{3 / 2} .
$$


Letting $s=q v^{2}, \quad J(q)=(2 \pi / q)^{1 / 2} \int_{0}^{1}\left(1-\exp \left[-q v^{2} / 2\right]\right) d v / v^{2} . \quad$ By $I(p)=2+(2 / \pi)^{1 / 2}|p| J\left(p^{2}\right)$, so that

$$
I(p)=2+2 \int_{0}^{1}\left(1-\exp \left[-p^{2} v^{2} / 2\right]\right) d v / v^{2} .
$$

Then, obviously, $I(p) \geqq 2$, and

$$
I(p) \leqq 2+2 \int_{0}^{\infty}\left(1-\exp \left[-p^{2} v^{2} / 2\right]\right) d v / v^{2}=2+(2 \pi)^{1 / 2}|p|
$$

by a second use of (4.8).

The following theorem will enable us to find the proper order of $s$ in certain special cases. Let $A_{n}=\sum_{j=0}^{n}\left|c_{j}\right|$ and $B_{n}=\sum_{j-0}^{n} j\left|c_{j}\right|$.

Theorem 6.2. If (6.19) is satisfied, and if $0<\alpha<1$, then:

(1) If $s=\epsilon /(2(1+\alpha) \log \log n)^{1 / 2}$, m.e. $\left\{N_{n}(c+s x)\right\}$

$$
<\frac{1}{(\log n)^{1+\alpha}}\left[\frac{7+\log n}{2 \pi}+\frac{(2 \log \log n)^{1 / 2}}{\epsilon \pi^{1 / 2}}\left(A_{n}+B_{n}\right)\right] .
$$

(2) If $s=A_{n} /(2(1-\alpha) \log \log n)^{1 / 2}$,

$$
\text { m.e. }\left\{N_{n}(c+s x)\right\}>(1 / \pi)(\log n)^{\alpha-1} \int_{0}^{1} R_{n} d t .
$$

Proof. Using (6.2) and Lemma 6.2,

$$
\text { m.e. }\left\{N_{n}(c+s x)\right\}>(1 / \pi) \int_{0}^{1} R_{n} \exp \left[-X_{n}^{2}(c) / 2 s^{2}\right] d t \text {. }
$$

For $0 \leqq t \leqq 1$, we find, using (4.2), that $\left|X_{n}(c)\right|<A_{n}$. Thus

$$
\text { m.e. }\left\{N_{n}(c+s x)\right\}>(1 / \pi) \exp \left[-A_{n}^{2} / 2 s^{2}\right] \int_{0}^{1} R_{n} d t,
$$

and for $s=A_{n} /(2(1-\alpha) \log \log n)^{1 / 2}$, we obtain (6.21). On the other hand, Lemma 6.2 yields

m.e. $\left\{N_{n}(c+s x)\right\}<(1 / \pi) \int_{0}^{1} R_{n} \exp \left[-X_{n}^{2}(c) / 2 s^{2}\right] d t$

$$
\begin{gathered}
+\left(1 / s(2 \pi)^{1 / 2}\right) \int_{0}^{1} R_{n}\left|Y_{n}(c)\right| \exp \left[-X_{n}^{2}(c) / 2 s^{2}\right] d t \\
<\exp \left[-\epsilon^{2} / 2 s^{2}\right]\left[(1 / \pi) \int_{0}^{1} R_{n} d t+\left(1 / s(2 \pi)^{1 / 2}\right) \int_{0}^{1} R_{n}\left|Y_{n}(c)\right| d t\right] .
\end{gathered}
$$


Using (4.2), we see that for $0 \leqq t \leqq 1$,

$$
\left|Y_{n}(c)\right|<\left(K_{n} / D_{n}\right)^{1 / 2} B_{n}+L_{n} A_{n} /\left(K_{n} D_{n}\right)^{1 / 2}
$$

so that

$$
\begin{aligned}
\left(1 / s(2 \pi)^{1 / 2}\right) \int_{0}^{1} R_{n}\left|Y_{n}(c)\right| d t & \\
& <\left(1 / s(2 \pi)^{1 / 2}\right)\left[B_{n} \int_{0}^{1} \frac{d t}{\left(K_{n}\right)^{1 / 2}}+A_{n} \int_{0}^{1}\left(L_{n} / K_{n}^{3 / 2}\right) d t\right] .
\end{aligned}
$$

Now,

$$
\int_{0}^{1} \frac{d t}{\left(K_{n}\right)^{1 / 2}}<1
$$

and, since $d K_{n} / d t=2 L_{n}$,

$$
\int_{0}^{1} \frac{L_{n}}{K_{n}^{3 / 2}} d t=-\left[K_{n}^{-1 / 2}\right]_{0}^{1}<1
$$

Thus, using (6.16),

$$
\text { m.e. }\left\{N_{n}(c+s x)\right\}<\exp \left[-\epsilon^{2} / 2 s^{2}\right]\left[\frac{7+\log n}{2 \pi}+\frac{1}{s(2 \pi)^{1 / 2}}\left(A_{n}+B_{n}\right)\right]
$$

and with $s=\epsilon /(2(1+\alpha) \log \log n)^{1 / 2}$, we obtain (6.20).

It is now easy to verify that if $A_{n}$ is bounded and if $B_{n}=O(\log n)$ as $n \rightarrow \infty$-for example, if $c_{j}=j^{-2}$-the estimates (6.20) and (6.21) become respectively

and

$$
\text { m.e. }\left\{N_{n}(c+s x)\right\}<\frac{p_{1}(\log \log n)^{1 / 2}}{(\log n)^{\alpha}}
$$

$$
\text { m.e. }\left\{N_{n}(c+s x)\right\}>p_{2}(\log n)^{\alpha}
$$

where $p_{1}$ and $p_{2}$ are absolute constants. Thus, in this case, the proper order of $s$ is $1 /(\log \log n)^{1 / 2}$.

As a possible application of the foregoing results, let us pose the following question: Suppose we have an equation of the type (6.5), the coefficients of which are to be determined experimentally. With what precision should the observations of $c_{0}, \cdots, c_{n}$ be made to insure that the equation with the observed coefficients will, on the average, have the same number of real roots as has (6.5), the equation with the theoretical coefficients? The observed coefficients can be written in the form $c_{j}+s x_{j}, j=0,1, \cdots, n$, where $s x_{j}$ represents the error term, $x_{j}$ being a standard normally distributed random variable, and $s$ the precision or standard deviation. We can suppose furthermore that $x_{0}, \cdots, x_{n}$ are independent. Thus the observed equation is precisely (6.1), and an investigation of the above question is nothing more than 
an investigation of the conditions under which m.e. $\left\{N_{n}(c+s x)\right\}$ is near $N_{n}(c)$. The results of this section, particularly Theorem 6.1, offer a partial answer to the problem.

7. Some further remarks about the roots of random algebraic equations. We assume in this section that the coefficients $x_{0}, \cdots, x_{n}$ of equation (1.1) are standard normally distributed independent random variables. I am indebted to Professor Kac for suggesting the following lemma which is a generalization of his formula (1.3).

LEMMA 7.1. If neither $a$ nor $b$ is a root of (1.1) and if $t_{1}, \cdots, t_{k}$ are the real roots of (1.1) in the interval $(a, b)$, and if $\phi(t)$ is continuous in $a \leqq t \leqq b$, then

$$
\sum_{j=1}^{k} \phi\left(t_{j}\right)=\lim _{\epsilon \rightarrow 0}(1 / 2 \epsilon) \int_{a}^{b} \phi(t) \psi_{\epsilon}(F)|\partial F / \partial t| d t .
$$

Proof. Choose $\epsilon>0$ so small that no turning point of $F$ in $(a, b)$, which is not at the same time a zero of $F$, has absolute value less than $\epsilon$. Then if $S_{\epsilon}=E_{(t)}[|F|<\epsilon]$, we can write $S_{\epsilon}=\sum_{j=1}^{k} I_{j}$, where $I_{j}$ is an open interval. Then, since we obviously can suppose that $\phi$ is real,

$$
2 \epsilon \min _{t \in I_{j}} \phi(t)<\int_{I_{j}} \phi(t)|\partial F / \partial t| d t<2 \epsilon \max _{t \in I_{j}} \phi(t),
$$

so that

$$
\sum_{j=1}^{k} \min _{t \in I_{j}} \phi(t)<(1 / 2 \epsilon) \int_{S_{\epsilon}} \phi(t)|\partial F / \partial t| d t<\sum_{j=1}^{k} \max _{t \in I_{j}} \phi(t) .
$$

Letting $\epsilon \rightarrow 0$, the lemma follows from the continuity of $\phi$.

Formula (1.3) is obtained from Lemma 7.1 by taking $\phi(t) \equiv 1$. We find that

$$
\text { m.e. }\left\{\sum_{j=1}^{k} \phi\left(t_{j}\right)\right\}=\lim _{\epsilon \rightarrow 0}(1 / 2 \epsilon) \int_{a}^{b} \phi(t) \text { m.e. }\left\{\psi_{\epsilon}(F)|\partial F / \partial t|\right\} d t
$$

or

$$
\text { m.e. }\left\{\sum_{j=1}^{k} \phi\left(t_{j}\right)\right\}=(1 / \pi) \int_{a}^{b} \phi(t) R_{n} d t .
$$

To prove (7.1), we follow exactly the method used by $\mathrm{Kac}$ in the case $\phi(t) \equiv 1[5$, pp. 316-318]. We need only to remark that $\phi(t)$ is bounded for $a \leqq t \leqq b$, and that the probability that either $a$ or $b$ is a root of (1.1) is zero. In case the resulting integral exists, we can put $a=-\infty$ and $b=+\infty$ in (7.2).

Formula (1.3) shows [5, p. 320] that, on the average, the real roots of (1.1) cluster about 1 and -1 . The following theorem gives an indication of just how close the roots are to these points. It states, in effect, that in finding the average sum of the real roots, we obtain the correct asymptotic result by taking each positive root as 1 and each negative root as -1 . Recalling 
that the average number of roots in any open interval containing 1 and -1 is asymptotic to $(2 / \pi) \log n$, we have

THEOREM 7.1. If $t_{1}, \cdots, t_{k}$ are the real roots of $(1.1)$ in the interval $(-A, A)$ with $A>1$, then m.e. $\left\{\sum_{j=1}^{k}\left|t_{j}\right|\right\} \sim(2 / \pi) \log n$.

Proof. Putting $\phi(t)=|t|$ in (7.2), we find

m.e. $\left\{\sum_{j=1}^{k}\left|t_{j}\right|\right\}=(2 / \pi) \int_{0}^{A} t R_{n} d t=(2 / \pi) \int_{0}^{1} t R_{n} d t+(2 / \pi) \int_{1 / A}^{1} R_{n} d t / t$.

We shall need the following estimates [5, pp. 319-320]:

$$
\begin{array}{lr}
R_{n} \leqq((2 n+1) /(1-t))^{1 / 2}, & 0 \leqq t<1, \\
R_{n}>\left(1-(n+1)^{2} a^{2 n}\right)^{1 / 2} /\left(1-t^{2}\right), & 0 \leqq t \leqq a<1 .
\end{array}
$$

From (6.16), we see that $\int_{0}^{1} t R_{n} d t<\int_{0}^{1} R_{n} d t<(7+\log n) / 2$, and by (7.3) and (6.15),

Thus

$$
\begin{aligned}
\int_{1 / A}^{1} R_{n} d t / t & \leqq \int_{1 / A}^{1-1 / n} \frac{d t}{t\left(1-t^{2}\right)}+\int_{1-1 / n}^{1} \frac{(2 n+1)^{1 / 2} d t}{(1-t)^{1 / 2}} \\
& <\left(7+\log \left(A^{2}-1\right)+\log n\right) / 2 .
\end{aligned}
$$

$$
\text { m.e. }\left\{\sum_{j=1}^{k}\left|t_{i}\right|\right\}<\left(2 \log n+14+\log \left(A^{2}-1\right)\right) / \pi \text {. }
$$

In order to obtain a lower estimate, let $\epsilon$ and $\alpha$ be arbitrary positive numbers less than 1 . Then by (7.4),

$$
\begin{aligned}
\int_{0}^{1} t R_{n} d t & >\int_{0}^{(1-n \alpha-1)^{1 / 2}} t R_{n} d t \\
& >\left(1-(n+1)^{2}\left(1-n^{\alpha-1}\right)^{n}\right)^{1 / 2} \int_{0}^{\left(1-n^{\alpha-1}\right)^{1 / 2}} \frac{t d t}{1-t^{2}},
\end{aligned}
$$

and for $n$ sufficiently large, this can be made larger than

$$
(1-\epsilon)^{1 / 2} \int_{0}^{\left(1-n^{\alpha-1}\right)^{1 / 2}} \frac{t d t}{1-t^{2}} .
$$

Thus $\int_{0}^{1} t R_{n} d t>(1-\epsilon)^{1 / 2}(1-\alpha)(\log n) / 2$. On the other hand,

$$
\begin{aligned}
\int_{1 / A}^{1} R_{n} d t / t & >\int_{1 / A}^{1} R_{n} d t \\
& >(1-\epsilon)^{1 / 2}(1-\alpha)(\log n) / 2-\log ((A+1) /(A-1))^{1 / 2},
\end{aligned}
$$

following [5, p. 320]. Therefore 
m.e. $\left\{\sum_{j=1}^{k}\left|t_{j}\right|\right\}>(2 / \pi)(1-\epsilon)^{1 / 2}(1-\alpha) \log n-(1 / \pi) \log [(A+1) /(A-1)]$.

Since $\epsilon$ and $\alpha$ can be made arbitrarily small, the theorem follows at once.

A natural question to ask now is whether the average of the sum of the roots in an interval not containing 1 or -1 has a limit as $n \rightarrow \infty$. This question can be answered in the affirmative by means of the next theorem.

THEOREM 7.2. If $t_{1}, \cdots, t_{k}$ are the real roots of $(1.1)$ in the interval $(-a, a)$ with $0<a<1$, then

$$
\lim _{n \rightarrow \infty} \text { m.e. }\left\{\sum_{j=1}^{k}\left|t_{j}\right|\right\}=-(1 / \pi) \log \left(1-a^{2}\right) .
$$

Proof. From (7.2) and (6.15),

$$
\text { m.e. } \begin{aligned}
\left\{\sum_{j=1}^{k}\left|t_{j}\right|\right\} & =(2 / \pi) \int_{0}^{a} t R_{n} d t \\
& \leqq(2 / \pi) \int_{0}^{a} \frac{t d t}{1-t^{2}} \\
& =-(1 / \pi) \log \left(1-a^{2}\right)
\end{aligned}
$$

Using (7.4),

$$
\text { m.e. } \begin{aligned}
\left\{\sum_{j=1}^{k}\left|t_{j}\right|\right\} & >(2 / \pi)\left(1-(n+1)^{2} a^{2 n}\right)^{1 / 2} \int_{0}^{a} \frac{t d t}{1-t^{2}} \\
& =-(1 / \pi)\left(1-(n+1)^{2} a^{2 n}\right)^{1 / 2} \log \left(1-a^{2}\right) .
\end{aligned}
$$

Sincè $0<a<1, \lim _{n \rightarrow \infty}(n+1) a^{n}=0$, and our result follows.

\section{REFERENCES}

1. P. Appell and J. Kampé de Fériet, Fonctions hypergéometriques et hypersphériques, Paris, 1926.

2. H. Cramér, Random variables and probability distributions, Cambridge, 1937.

3. H. Hahn, Über die Darstellung gegebener Functionen durch singuläre Integrale, Denkschriften der K. Akademie der Wissenschaften, Vienna, Mathematisch-Naturwissenschaftliche Klasse, vol. 93 (1917) pp. 585-655. 464.

4. E. Hille, $A$ class of reciprocal functions, Ann. of Math. (2) vol. 27 (1925-1926) pp. 427-

5. M. Kac, On the average number of real roots of a random algebraic equation, Bull. Amer. Math. Soc. vol. 49 (1943) pp. 314-320.

CORNELL University, ITHACA, N. Y. 